\title{
Positive control of a minimal model of tumor growth with bevacizumab treatment*
}

\author{
Dániel András Drexler, Johanna Sápi, Levente Kovács
}

\begin{abstract}
Application of model-based control strategies for physiological control problems, like tumor therapy, may greatly improve the efficiency of clinical treatments. Our aim is to design a positive path tracking controller for a minimal model of tumor growth under angiogenic inhibition with bevacizumab treatment. We extend the dynamics of the system to ensure the positivity of the input, and design path tracking controller after exact linearization is carried out for the extended system. We carry out simulations for different reference signals for the path tracking controllers, and confirm that the tumor can be eliminated using bevacizumab therapy with physiologically feasible input signals. The resulting inputs are positive, and their maximal values are in physiologically feasible regions, thus the results may be applicable in clinical practice.
\end{abstract}

\section{INTRODUCTION}

The dosage of drugs in clinical treatments in practice is usually specified heuristically by physicians with appropriate expertise. However, these heuristic methods lack mathematical model-based background, thus one can not ensure that they yield the optimal solution [1]. Therefore, there may be potential benefits for clinical practice arising from modelbased design of clinical treatments.

There are different tumor treatment methods based on the physiological background of the therapy, the most commonly known therapies are chemotherapy and radiotherapy, while antiangiogenic therapy [1] is a less known targeted molecular therapy that has much less side-effect than the treatments mentioned previously. Antiangiogenic therapy uses angiogenic inhibitors as drugs: the drug inhibits angiogenesis [2], i.e. the formation of new blood vessels the tumor needs in order to be able to grow [3], this way inhibits tumor grows indirectly (and may lead to tumor regression as well). Angiogenic therapy was first used in combination with other therapies, however its application as monotherapy is being considered recently. In this work, we design antiangiogenic treatment using a model-based methodology.

The most known model for tumor growth under angiogenic inhibition was proposed by Hahnfeldt and his collaborates in 1999 [4]. This is a second-order model that describes dynamics with nonlinear ordinary differential equations, the

*This project has received funding from the European Research Council (ERC) under the European Unions Horizon 2020 research and innovation programme (grant agreement No 679681). D. A. Drexler was also supported by a Marie Curie International Research Staff Exchange Scheme Fellowship within the 7th European Community Framework Programme, FP7-PEOPLE2012-IRSES-316338.

The authors are with the Physiological Controls Research Center, Research and Innovation Center of Óbuda University, Óbuda University, Budapest, Hungary drexler.daniel@nik.uni-obuda.hu, sapi.johanna@nik.uni-obuda.hu, kovacs.levente@nik.uni-obuda.hu two equations describe the dynamics of the tumor volume and the dynamics of the supporting vasculature (i.e. the blood vessels that provide nutrients for the tumor). The model was identified based on experiments carried out on mice with three different inhibitors. However, these inhibitors are still very expensive, and the Hahnfeldt-model has undesirable nonlinear components. We use a minimal model here that was identified based on mice experiments [5], [6] using bevacizumab as inhibitor that is much cheaper and more available than the inhibitors used in [4].

The minimal model proposed in [6] is a second-order model with linear terms in its differential equation and one bilinear term. This is the simplest model that describes the dynamics of tumor growth and fits well for the measurements. The model describes the dynamics of tumor growth and the dynamics of the inhibitor (also called pharmakokinetics in some literature, see e.g. [7]). The minimal model is explained in Section II. Parametric identification and validation of the results is done in [6]. We use the acquired parameters here to design nonlinear path tracking controller with ensured positive control signal.

The input of the therapy is the rate of injection of the inhibitor that is always positive, since taking out drug from a patient is circumstantial and thus not available in practice. Hence, ensuring positivity of the control input in the level of the design is crucial. Ensuring positivity of the input can be done by applying a saturation on the control signal as it was done e.g. in [8], [9], however this modifies the model of the closed-loop system thus model-based techniques become less reliable. Here we extend the dynamics of the system with a fictive dynamics for the input signal that ensures positivity as explained in Section III.

The extended system is linearized using exact linearization, and finally a path tracking control law is given for the linearized system in Section III. The extended tumor model can be transformed to a series of integrators in its whole domain, thus it is equivalent to a series of integrators. The dynamical extension that is used to ensure positivity of the input is finally added to the controller. This control architecture ensures that the closed-loop system has path tracking ability, thus it can track many different reference signals providing great flexibility in the design of therapeutic protocols. In Section IV we analyze the closed-loop system with different reference signals based on simulations.

Most of the previous works in this field are based on the Hahnfeldt-model [4]. In [8], [9] linear optimal and robust controllers are designed for the Hahnfeldt-model linearized in an operation point with the positivity of the input being 
ensured using saturation on the control input. A modified Hahnfeldt-model (that also incorporates chemotherapy) is used in [10] and a combined therapy is designed using a combination of angiogenic inhibitors and cytotoxic agents as control inputs. In [11] optimal bang-bang control is designed for a tumor model with cytotoxic agent as input (i.e. the model describes chemotherapy). Optimal control of angiogenic therapy was considered in [12], where the authors design a control law to minimize a performance index based on a modified Hahnfeldt-model proposed in [13].

The results here use the minimal model proposed in [6], and ensure path tracking ability of the closed-loop system. The simulations are carried out with prescribed exponential decrease of the tumor volume with different half-lives for the tumor, the results are discussed in Section IV. The resulting control inputs are physiologically acceptable, i.e. they are positive and their maximal value is tolerable by the patient. The closed-loop system given here may help clinicians to design therapeutic protocols for angiogenic therapy.

\section{MINIMAL MODEL OF TUMOR GROWTH}

A minimal model of tumor growth with angiogenic inhibition [6] is described by the differential equations

$$
\begin{aligned}
\dot{x} & =a x-b x y \\
\dot{y} & =-c y+I
\end{aligned}
$$

where $x$ is the function that assigns for each positive time a tumor volume measured in $\mathrm{mm}^{3}, y$ is the function that assigns for each positive time an inhibitor serum level measured in $\mathrm{mg} / \mathrm{kg}$, while $I$ is the function that assigns for each positive time the inhibitor injection rate measured in $\mathrm{mg} /(\mathrm{kg} \cdot$ day $)$ (a unit means one $\mathrm{mg}$ of inhibitor per body mass $\mathrm{kg}$ of the host each day). The parameters of the model are the tumor growth rate parameter $a$ measured in $1 /$ day, the inhibition rate parameter $b$ measured in $\mathrm{kg} /(\mathrm{mg} \cdot$ day) and the clearance of the inhibitor $c$ measured in $1 /$ day.

The values of the parameter are acquired based on experiments with $\mathrm{C} 57 \mathrm{Bl} / 6$ mice using bevacizumab as inhibitor and C38 colon adenocarcinoma as tumor [5]. After parametric identification in [6], the values of the parameters are $a=$ $0.271 /$ day and $b=0.0072 \mathrm{~kg} /(\mathrm{mg} \cdot$ day $)$. We use the value $c=\ln (2) / 3.91 /$ day acquired from mice experiments in [7].

The nontrivial equilibrium points of the model are acquired by solving

$$
\begin{aligned}
& 0=a x_{\infty}-b x_{\infty} y_{\infty} \\
& 0=-c y_{\infty}+I_{\infty}
\end{aligned}
$$

that results in

$$
\begin{aligned}
y_{\infty} & =\frac{a}{b} \\
I_{\infty} & =c \frac{a}{b}
\end{aligned}
$$

that are independent of the tumor volume. Using the parameters from [6] this means that we need

$$
y_{\infty}=36.48 \frac{\mathrm{mg}}{\mathrm{kg}}
$$

amount of inhibitor if we want to maintain constant tumor volume; otherwise we need higher inhibitor level if we want to decrease the tumor volume, and lower inhibitor level if we want to increase the tumor volume. The required injection rate related to the equilibrium inhibitor level is

$$
I_{\infty}=6.48 \frac{\mathrm{mg}}{\mathrm{kg} \cdot \text { day }} \text {. }
$$

\section{POSITIVE PATH TRACKING CONTROL}

The control input of the model is the rate of inhibitor injection, that needs to be positive, since we can not remove inhibitor from the host. Thus we need to assure that the rate of inhibition is positive. We achieve this by extending the dynamics of the original system with the differential equation

$$
\dot{I}=-I u
$$

that has the solution

$$
I(t)=I(0) e^{\int_{0}^{t} u(\tau) d \tau}
$$

that has the same sign as $I(0)$. Therefore, if $I(0)>0$, then $I(t)>0$ for all positive time regardless of the sign of the function $u$. In what follows, we extend the dynamics of the tumor model with (9), and design controller for the extended system with the input function $u$. Thus the output of the designed controller is $u$ that can have positive and negative values as well. In the implementation process, we add the dynamical extension (9) to the designed controller (see Fig. 1 ), thus the output of the extended controller will be positive due to (10).

The extended model can be described by the differential equation

$$
\underline{\dot{x}}=f(\underline{\mathrm{x}})+g(\underline{\mathrm{x}}) u
$$

with $\underline{\mathrm{x}}=(x, y, I)^{\top}$, and the vector fields $f$ and $g$ given by

$$
f(\underline{\mathrm{x}})=\left(\begin{array}{c}
a x-b x y \\
-c y+I \\
0
\end{array}\right), \quad g(\underline{\mathrm{x}})=\left(\begin{array}{c}
0 \\
0 \\
-I
\end{array}\right) .
$$

The output of the system is the tumor volume, thus it is described by the scalar field $h=x$. We design path tracking control using exact linearization [14]. The third-order extended system is transformed to a series of integrators by differentiating the scalar field $h$ (i.e. the output of the system) three times. The first derivative of $h$ is

$$
\dot{h}=h^{\prime} \underline{\dot{x}}=h^{\prime} f(\underline{\mathrm{x}})+h^{\prime} g(\underline{\mathrm{x}}) u:=L_{f} h+L_{g} h u
$$

where $L_{f} h$ is the Lie-derivative [14] of the scalar field $h$ along the vector field $f$ defined as $L_{f} h=h^{\prime} f$, while $L_{g} h$ is the Lie-derivative of the scalar field $h$ along the vector field $g$ defined as $L_{g} h=h^{\prime} g$. The values of the Lie-derivatives are

$$
\begin{aligned}
L_{f} h & =a x-b x y \\
L_{g} h & =0
\end{aligned}
$$

so the first derivative of the output does not depend on $u$ since $L_{g} h=0$. The second derivative of the output is the function

$$
\begin{aligned}
\ddot{h}= & \left(h^{\prime} f(\underline{\mathrm{x}})\right)^{\prime} \underline{\dot{\mathrm{x}}}=\left(h^{\prime} f(\underline{\mathrm{x}})\right)^{\prime} f(\underline{\mathrm{x}})+\left(h^{\prime} f(\underline{\mathrm{x}})\right)^{\prime} g(\underline{\mathrm{x}}) u \\
& :=L_{f}^{2} h+L_{g} L_{f} h u
\end{aligned}
$$


where $L_{f}^{2} h$ is the Lie-derivative of the scalar field $L_{f} h$ along the vector field $f$, while $L_{g} L_{f} h$ is the Lie-derivative of the scalar field $L_{f} h$ along the vector field $g$. The values of the Lie-derivatives are

$$
\begin{aligned}
L_{f}^{2} h & =x\left((a-b y)^{2}-b(I-c y)\right) \\
L_{g} L_{f} h & =0
\end{aligned}
$$

thus the second derivative of the output does not depend on $u$ since $L_{g} L_{f} h=0$. The third derivative of the output is similarly

$$
h^{(3)}=L_{f}^{3} h+L_{g} L_{f}^{2} h u
$$

where $L_{f}^{3} h$ is the Lie-derivative of the scalar field $L_{f}^{2} h$ along the vector field $f$, while $L_{g} L_{f}^{2} h$ is the Lie-derivative of the scalar field $L_{f}^{2} h$ along the vector field $g$. The values of the Lie-derivatives are

$$
\begin{aligned}
L_{f}^{3} h= & x((b c-2 b(a-b y))(I-c y))+ \\
& +x\left(\left((a-b y)^{2}-b(I-c)\right)(a-b y)\right) \\
L_{g} L_{f}^{2} h= & b x I
\end{aligned}
$$

thus the third derivative of the output depends on the input if $x \neq 0$ and $I \neq 0$, since in this case $L_{g} L_{f}^{2} h$ is not zero. The system is equivalent to the series of integrators with state variables $z_{1}=x, z_{2}=\dot{x}$ and $z_{3}=\ddot{x}$

$$
\begin{aligned}
& \dot{z}_{1}=z_{2}\left(=L_{f} h\right) \\
& \dot{z}_{2}=z_{3}\left(=L_{f}^{2} h\right) \\
& \dot{z}_{3}=v\left(=L_{f}^{3} h+L_{g} L_{f}^{2} u\right)
\end{aligned}
$$

everywhere except $x=0$ or $I=0$ with a new fictive input $v$, thus path tracking control can be designed for this series of integrators that results in the control law for $v$ which can be transformed to the input of the extended system from (24) as

$$
u=\frac{v-L_{f}^{3} h}{L_{g} L_{f}^{2} h} .
$$

Let the reference output and its derivatives up to the third order be defined by $x_{r e f}, \dot{x}_{r e f}, \ddot{x}_{r e f}, x_{r e f}^{(3)}$, then the path tracking control law for the series of integrators is

$$
v=k_{0}\left(x_{r e f}-z_{1}\right)+k_{1}\left(\dot{x}_{r e f}-z_{2}\right)+k_{2}\left(\dot{x}_{r e f}-z_{3}\right)+x_{r e f}^{(3)}
$$

that ensures that the path tracking error dynamics is governed by the differential equation

$$
0=e^{(3)}+k_{2} \ddot{e}+k_{1} \dot{e}+k_{0} e
$$

with $e=x_{r e f}-z_{1}=x_{r e f}-x$. Thus, the control law for control input $u$ expressed with the states of the system is

$u=\frac{k_{0}\left(x_{r e f}-h\right)+k_{1}\left(\dot{x}_{r e f}-L_{f} h\right)+k_{2}\left(\dot{x}_{r e f}-L_{f}^{2} h\right)+x_{r e f}^{(3)}-L_{f}^{3} h}{b x I}$.

The block diagram of the closed loop system is in Fig. 1.

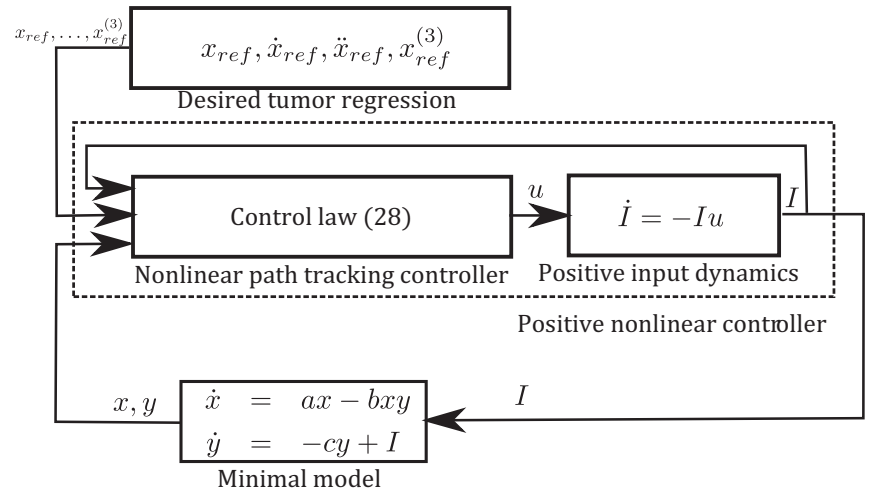

Fig. 1. The block diagram of the closed-loop system with the reference signal, positive nonlinear path tracking controller and the minimal model

\section{SIMULATION RESULTS}

Simulations were carried out using the parameters given in Section II, with initial tumor volume $x(0)=10000 \mathrm{~mm}^{3}$ and initial inhibitor level $y(0)=0.01 \mathrm{mg} / \mathrm{kg}$. The gain parameters in the control law were $k_{0}=1, k_{1}=3, k_{2}=3$, so the characteristic equation of the error dynamics has the roots -1 with multiplicity of three, thus the tracking error is stable and converges to zero.

The reference tumor volume and its derivatives were chosen such that the tumor volume is eliminated exponentially, i.e.

$$
\begin{aligned}
& x_{\text {ref }}=(x(0)-1) e^{-t /\left(\ln (2) T_{\text {hal }}\right)} \\
& \dot{x}_{\text {ref }}=(x(0)-1) e^{-t /\left(\ln (2) T_{\text {hal }}\right)}\left(-1 /\left(\ln (2) T_{\text {hal }}\right)\right) \\
& \ddot{x}_{\text {ref }}=(x(0)-1) e^{-t /\left(\ln (2) T_{\text {hal }}\right)}\left(-1 /\left(\ln (2) T_{\text {hal }}\right)\right)^{2}(31) \\
& x_{\text {ref }}^{(3)}=(x(0)-1) e^{-t /\left(\ln (2) T_{\text {hal }}\right)}\left(-1 /\left(\ln (2) T_{\text {hal }}\right)\right)^{3}(32)
\end{aligned}
$$

where $T_{\text {half }}$ is the prescribed half-life of the tumor. Simulations were carried out with half-lives $T_{\text {half }} \in\{5,15,25,35\}$ days, the treatments were 200 days long in each case, the results are in Figs. 2-4.

After an initial transient, the tumor volumes starts to decrease according to the prescribed exponential trend, as it can be observed in Fig. 2. At the beginning of the treatment, the tumors grow, but after the inhibitor level exceeds the limit given by (7) (see Fig. 3), the tumor volumes start to decrease. The tumor volumes tend to the prescribed equilibrium state $x_{\infty}=1 \mathrm{~mm}^{3}$ (this is not zero, since the tumor can not be eliminated totally using antiangiogenic therapy due to physiological reasons).

The inhibitor levels increase rapidly in the initial transient (see Fig. 3), and after the transients they tend to the equilibrium value given by (7). Note that the inhibitor level is always over this limit (or equal to that) during the therapy (except at the beginning, because the initial value of the inhibitor level is below this limit), so the tumor volume decreases (or stagnates) during the therapy.

The inhibitor injection rates are in Fig. 4. At the beginning of the treatment, there are high amount of injections in order to increase the inhibitor level over the limit (7). After the inhibitor level has been increased, there is a rest period (i.e. 


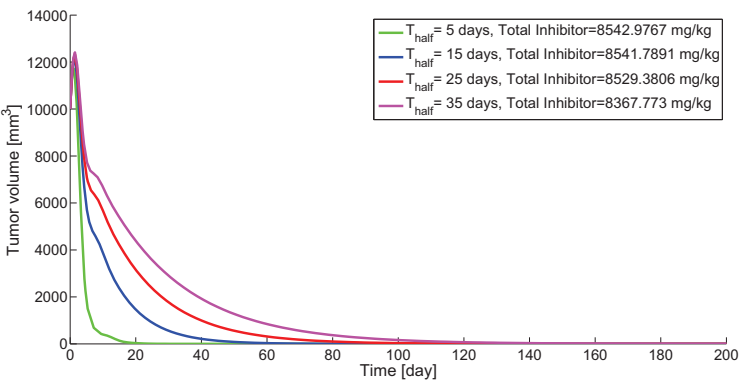

Fig. 2. The tumor volumes during 200 days long therapies with different prescribed tumor volume half-lives

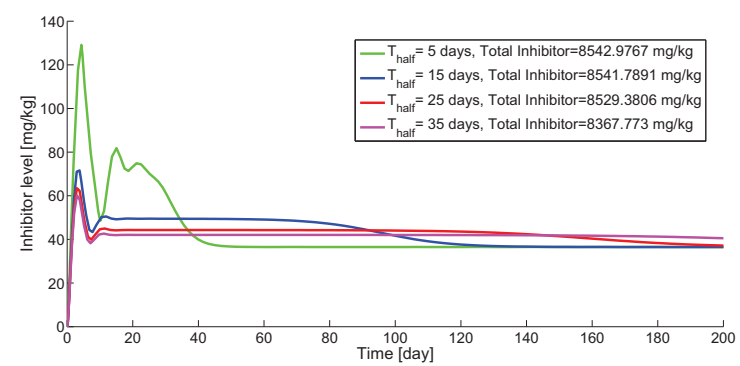

Fig. 3. The inhibitor levels during 200 days long therapies with different prescribed tumor volume half-lives

there are no injections) while the inhibitor levels decrease. Advantages of intermittent drug administration is discussed in details in [15]. After some transients the inhibitor injection rates tend to the equilibrium value given by (8).

The total amount of inhibitors used during the therapy are in the legends of Figs. 2-4. These values are between $8367 \mathrm{mg} / \mathrm{kg}$ and $8542 \mathrm{mg} / \mathrm{kg}$. The difference between the used inhibitor amounts is not significant. The appropriate choice of the reference signal for the control system may be chosen by finding physiologically suitable protocols from Fig. 4.

\section{CONCLUSION}

Path tracking controller was designed for the minimal model of tumor growth using the easily accessible bevacizumab as angiogenic inhibitor. Simulations of the closedloop system showed that the required drug inputs for the desired tumor regression are physiologically feasible, thus

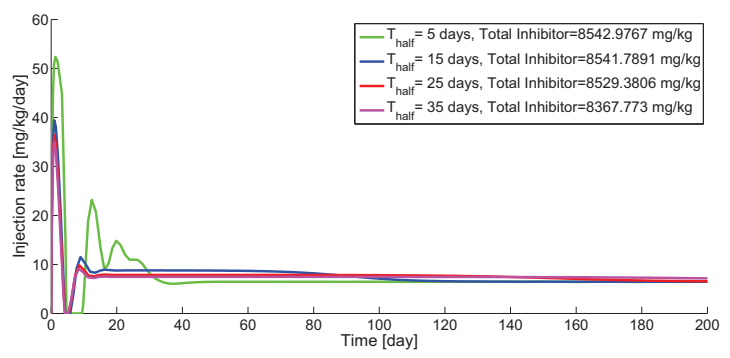

Fig. 4. The inhibitor injection rates during 200 days long therapies with different prescribed tumor volume half-lives the protocols resulted from the simulations may be used in the practice. The applicability of the results may be further increased by extending the controller with robust techniques used e.g. in [9], [16].

\section{REFERENCES}

[1] O. Distler, M. Neidhart, R. E. Gay, and S. Gay, "The molecular control of angiogenesis," International Reviews of Immunology, vol. 21, no. 1, pp. 33-49, 2002.

[2] N. Ferrara, "Vascular endothelial growth factor and the regulation of angiogenesis," Recent Progress in Hormone Research, vol. 55, pp. $15-35,2000$.

[3] J. H. Distler, A. Hirth, M. Kurowska-Stolarska, R. E. Gay, S. Gay, and O. Distler, "Angiogenic and angiostatic factors in the molecular control of angiogenesis," The Quarterly Journal of Nuclear Medicine, vol. 47, no. 3, pp. 149-161, 2003.

[4] P. Hahnfeldt, D. Panigrahy, J. Folkman, and L. Hlatky, "Tumor development under angiogenic signaling: A dynamical theory of tumor growth, treatment response, and postvascular dormancy," Cancer Research, vol. 59, pp. 4770-4775, 1999.

[5] J. Sápi, L. Kovács, D. A. Drexler, P. Kocsis, D. Gajári, and Z. Sápi, "Tumor volume estimation and quasi-continuous administration for most effective bevacizumab therapy," PLoS ONE, vol. 10, no. 11, pp. $1-20,112015$.

[6] D. A. Drexler, J. Sápi, and L. Kovács, "A minimal model of tumor growth with angiogenic inhibition using bevacizumab," in Proceedings of the 2017 IEEE 15th International Symposium on Applied Machine Intelligence and Informatics, accepted for publication, 2017.

[7] F. Wu, M. Tamhane, and M. Morris, "Pharmacokinetics, lymph node uptake, and mechanistic pk model of near-infrared dye-labeled bevacizumab after iv and sc administration in mice," The AAPS Journal, vol. 14 , no. 2,2012

[8] J. Sápi, D. A. Drexler, I. Harmati, Z. Sápi, and L. Kovács, "Qualitative analysis of tumor growth model under antiangiogenic therapy: choosing the operating point and design parameters for controller design," Optimal Control Applications \& Methods, p. 19, 2015.

[9] L. Kovács, A. Szeles, J. Sápi, D. A. Drexler, I. Rudas, I. Harmati, and Z. Sápi, "Model-based angiogenic inhibition of tumor growth using modern robust control method," Computer Methods and Programs in Biomedicine, vol. 114, pp. e98-e110, 2014.

[10] J. Klamka, H. Maurer, and A. Swierniak, "Local controllability and optimal control for a model of combined anticancer therapy with control delays," Mathematical Biosciences and Engineering, vol. 14, no. 1, pp. 195-216, 2017.

[11] F. S. Lobato, V. S. Machado, and V. Steffen, "Determination of an optimal control strategy for drug administration in tumor treatment using multi-objective optimization differential evolution," Computer Methods and Programs in Biomedicine, vol. 131, pp. 51 - 61, 2016.

[12] N. Nath, T. Burg, D. M. Dawson, and E. Iyasere, "Optimizing antiangiogenic therapy for tumor minimization," in American Control Conference (ACC), 2010, June 2010, pp. 1242-1247.

[13] A. d'Onofrio and A. Gandolfi, "Tumor eradication by antiangiogenic therapy: analysis and extensions of the model by hahnfeldt et al (1999)," Mathematical Biosciences, vol. 191, no. 2, pp. 159-184, 2004.

[14] A. Isidori, Nonlinear Control Systems. Springer-Verlag London, 1995.

[15] J. Sápi, D. A. Drexler, and L. Kovács, "Comparison of protocol based cancer therapies and discrete controller based treatments in the case of endostatin administration," in In Proceedings of the 2016 IEEE International Conference on Systems, Man, and Cybernetics, 2016, pp. 3830-3835.

[16] J. Sápi, D. A. Drexler, and L. Kovács, "Parameter optimization of $\mathrm{H}_{\infty}$ controller designed for tumor growth in the light of physiological aspects," in 14th IEEE International Symposium on Computational Intelligence and Informatics, 2013, pp. 19-24. 